\title{
Undecidability and Incompleteness in Classical Mechanics
}

\author{
N. C. A. da Costa $^{1}$ and F. A. Doria ${ }^{2,3}$ \\ Received October 2, 1990
}

\begin{abstract}
We describe Richardson's functor from the Diophantine equations and Diophantine problems into elementary real-valued functions and problems. We then derive a general undecidability and incompleteness result for elementary functions within ZFC set theory, and apply it to some problems in Hamiltonian mechanics and dynamical systems theory. Our examples deal with the algorithmic impossibility of deciding whether a given Hamiltonian can be integrated by quadratures and related questions; they lead to a version of Gödel's incompleteness theorem within Hamiltonian mechanics. A similar application to the unsolvability of the decision problem for chaotic dynamical systems is also obtained.
\end{abstract}

\section{INTRODUCTION}

The first example of a sensible mathematical statement which is undecidable with respect to an accepted axiomatic system was given through the 19th century proof of the independence of Euclid's parallel postulate from the remaining axioms of plane geometry. That independence proof is certainly one of the greatest technical and conceptual achievements of the last century's mathematics, since it emphasizes the formal nature of an axiomatic system through the separation between syntaxis and semantics, and shows that a naively-looking, intuitively "true" geometrical assertion can be unprovable from "natural" first principles.

However, we had to wait until 1931 for another major progress in that direction. As is well known, Gödel (1931) showed in a famous paper that a still more basic axiom system, that of formalized arithmetic, also contains

\footnotetext{
'Institute for Advanced Studies, University of São Paulo, 05508 São Paulo SP Brazil.

${ }^{2}$ IMSSS/Stanford University, Stanford, California 94305-4115.

${ }^{3}$ Permanent address: Center for the Study of Mathematical Theories of Communication, IDEA/School of Communications, Federal University of Rio de Janeiro, 22290 Rio de Janeiro RJ Brazil.
} 
undecidable statements, that is, sentences that can neither be proved nor disproved with the tools available within the formal system. However, since the example that Gödel gave for an undecidable arithmetic statement was quite weird and without an obvious explicit mathematical meaning, it was hoped at that time that the undecidability phenomenon (and examples of undecidable sentences) would remain a subject far from everyday mathematical activity, despite the fact that the precedent of plane geometry clearly indicates that sensible mathematical statements can be undecidable within a standard axiomatic system [a recent rigorous, albeit elementary discussion and proof of Gödel's result can be found in Uspensky (1987)].

Cohen's 1963 proof of the independence of the continuum hypothesis with respect to the Zermelo-Fraenkel set-theoretic axioms furnished a new important example of a sensible mathematical sentence that is undecidable within a powerful axiomatic system (Cohen, 1966); it has been frequently pointed out that Cohen's proof is in many respects similar to the 19th century proof of the independence of the parallel postulate.

As it had long been known that the continuum hypothesis has immediate relevance to several problems in point-set topology, measure theory, and analysis (Şierpiński, 1956), from 1963 onward undecidable statements within the Zermelo-Fraenkel system plus the full axiom of choice have been creeping up in all branches of mathematics. For a review of the chief results see Dales and Woodin (1987) and Kunen and Vaught (1984).

So much for independence results (and the corresponding undecidable sentences) in mathematics; undecidability is everywhere. And how about physics? We give in the present paper several examples of very simple and yet powerful undecidability and incompleteness results in classical mechanics and in dynamical systems theory. They all stem from Richardson's (1968) proof that the theory of elementary functions in classical analysis is undecidable. Elementary functions are those that belong to an algebra $\mathscr{A}$ that contains the rational numbers, polynomials, sines and cosines, exponentials, plus the number $\pi$, and is closed under sum, product, and function composition. Richardson's main result translates Diophantine equations into elementary functions, and allows us to obtain all the undecidability and incompleteness consequences of Hilbert's Tenth Problem inside $\mathscr{A}$ when $\mathscr{A}$ is seen as embedded into a sufficiently powerful axiom system, such as ZFC (Davis $e t$ al., 1976). Therefore, innocent-looking problems in analysis are seen to be intractable because they are equivalent to the solution of an intractable Diophantine equation.

Moreover, as the undecidability of Hilbert's Tenth Problem is a direct consequence of Gödel's theorem, our results are not tied to a particular axiomatic system; they are essentially examples of undecidability and incompleteness results in classical analysis within a sufficiently rich axiom system 
$T$ (whose properties are detailed below) that can be extended to undecidability and incompleteness results in axiomatized formulations of physics within $T$ or within one of its extensions. This means that as long as our mathematical (and axiomatic) tools allow us to formalize algorithmicity, which means recursive function theory, we will get the spector of undecidability and incompleteness in our theory.

Section 2 reviews concepts like "undecidability" and "incompleteness" within formal systems. Section 3 describes the construction of Richardson's functor from Diophantine equations (and exponential Diophantine equations) into the algebra of elementary functions of a real variable. We conclude the section with a "general incompleteness theorem" that serves as a blueprint for the results we obtain in classical mechanics.

Sections 4 and 5 apply those ideas to Hamiltonian dynamics and to chaotic dynamical systems. Our main undecidability and incompleteness results deal with the integrability of Hamilton-Jacobi equations, and with the possibility of proving that a given dynamical system has a chaotic behavior.

Finally, in Section 6 we evaluate our results and try to make some sense out of such a seemingly confusing plethora of undecidability and incompleteness results for the mathematics of an empirical science like physics.

The problems that we discuss here have been around for some time. See on the integrability problem Lichtenberg and Lieberman (1983) and Tabor (1989). On the decision problem for chaotic systems ("if we look at the system's equations, can we tell whether it is chaotic?") see Hirsch (1985). A previous discussion on the relation between Gödel's incompleteness theorem and physics can be found in Chaitin (1982). A particular example of a dynamical system with undecidable properties has been given by Moore (1990).

\section{NOTATION AND BACKGROUND}

We suppose that our discussion takes place within an axiomatic framework flexible enough to include from arithmetic to calculus on manifolds and the geometry of symplectic structures. A detailed description of one such axiomatization within Zermelo-Fraenkel set theory can be found in da Costa and Doria (1990a); we also quote as related work our papers (da Costa and Doria, 1990b, 1991; da Costa et al., 1990). The concepts we require are described below.

However, we note that since we will be mainly discussing algorithmic impossibilities, we are not tied to a particular axiom system; it is enough to know that the main objects we must handle in our proofs can be adequately formalized within some axiomatics. 
Our notation is standard. We use a few logical symbols, such as $\forall$ ("for all"), $\exists$ ("there exists"), $\wedge$ ("and"), $\vee$ ("or"), $\rightarrow$ ("if, ... then"), $\leftrightarrow$ ("if and only if"). $T \vdash \xi$ means, "the sentence $\xi$ can be deduced from the axioms of the theory $T$." $\mathbf{M}=\xi$ means, " $\xi$ is true for the model M." The arrow $\rightarrow$ will also denote maps; meaning will be clear from the context.

We denote the set of natural numbers by $\omega_{0}$; the integers are $Z$, the rationals are $Q$ and the reals are $R$.

\section{Algorithms, Effectiveness, and Formal Systems}

An algorithm is a mechanical procedure that generates finite sequences of symbols out of finite sequences of symbols. Intuitively, an algorithm satisfies the following guidelines:

1. We start from a finite sequence of symbols, the algorithm's input.

2. If and when the algorithm concludes its task, it produces another finite sequence of symbols, the algorithm's output.

3. The actual procedure to be followed can be explicitly written out as a finite sequence of symbols.

4. The computation proceeds in a step-by-step manner, and produces an output (if any) after a finite number of steps.

5. Intermediate computations can also be coded as a finite sequence of symbols.

6. The whole procedure is deterministic.

The theory of algorithms, or the theory of effective computability, is formalized in several different but equivalent ways: recursive function theory, the theory of Turing machines, the theory of Post's canonical languages, Markov algorithms, and so on. Church's Thesis asserts that each one of those formalizations adequately "translates" the intuitive concept of an algorithm. For detailed references see Machtey and Young (1978), Mendelson (1987), and Rogers (1967).

Let $\omega_{0}$ be the natural number sequence, and let $A \subseteq \omega_{0}$ be one of its (possibly infinite) subsets. We say that $A$ is recursive if there is an algorithm that allows us to answer, for every $n \in \omega_{0}$, the question "does $n \in A$ ?"

$A$ is recursively enumerable if there is an algorithm which, given as its input the sequence of all natural numbers $0,1,2, \ldots$, generates as its output a complete list of all the elements of $A$. That listing is not necessarily ordered by $<$, and repetitions are allowed.

Every recursive set is recursively enumerable, but there are recursively enumerable sets that are not recursive.

One can easily define concepts like "formal system" "formal theory," "axiomatizable theory," plus the semantic notions of "interpretation of [the 
language of] a theory," "truth," and the concept of "model" for a set of sentences in a theory [see, for instance, Manin (1977) and Mendelson (1987); a particular example is described in da Costa et al. (1990)]. Anyway, they are the standard ones.

We are going to deal here with a few specific formal systems. We denote by $L_{\mathrm{Ar}}^{1}$ the first-order language of arithmetic, and $L_{\mathrm{ZFC}}^{1} \supset L_{\mathrm{Ar}}^{1}$ is the firstorder language of Zermelo-Fraenkel's axiomatic set theory with the axiom of choice; the whole of "standard" or "classical" mathematics can be formalized within $L_{\mathrm{ZFC}}^{1}$, so that all usual mathematical theorems are theorems in $L_{\mathrm{ZFC}}^{1}$. References can be found in da Costa et al. (1990), Manin (1977), and Mendelson (1987).

In the general case, we will require of any formal theory $T$ that its language be a first-order language, and $L_{T}^{1} \supset L_{\mathrm{Ar}}^{1}$, plus some extra conditions that are stated below.

Given any such theory $T$, we suppose that there is an effective coding $\imath: \mathscr{S}_{T} \rightarrow \omega_{0}$ of the set of sentences of $T ; \imath$ is $1-1$ and onto, and as it is effective, given any $k \in \omega_{0}$, we can algorithmically obtain the corresponding sentence $\xi_{k} \in \mathscr{S}_{T}$.

We denote by $\mathscr{T}_{T} \supset \mathscr{S}_{T}$ the set of all theorems of $T$; for all theories that are discussed in the present paper, $\mathscr{T}_{T}$ is a recursively enumerable set.

We then state:

Definition 2.1. For any such theory $T$, we have:

1. If $\mathscr{T}_{T} \supset \mathscr{S}_{T}$ is a recursive subset, then $\mathscr{T}$ is a decidable theory.

2. If $\mathscr{T}_{T} \supset \mathscr{S}_{T}$ is not recursive, then $T$ is an undecidable theory.

3. If, for all sentences $\xi \in \mathscr{S}_{T}$, either $\xi \in \mathscr{T}_{T}$ or $(\neg \xi) \in \mathscr{T}_{T}$, we say that $T$ is a complete theory.

4. If there is a $\xi_{0} \in \mathscr{S}_{T}$ such that $\xi_{0} \notin \mathscr{T}_{T}$ as well as $\left(\neg \xi_{0}\right) \notin \mathscr{T}_{T}$, then $T$ is an incomplete theory, and $\xi_{0}$ is said to be an undecidable sentence of $T$.

Remark 2.2. We will frequently write $T \vdash \xi$ for $\xi \in \mathscr{T}_{T}$.

The next definition is discussed in Rogers (1967, pp. 319-321). Let $\mathscr{S}_{\mathrm{Ar}} \supset \mathscr{S}_{T}$ be the set of arithmetic sentences in $T$ :

Definition 2.3. If $L_{T}^{1} \supset L_{\mathrm{Ar}}^{1}$, then:

1. For $\zeta, \xi \in \mathscr{S}_{T}, \zeta$ and $\xi$ are $T$-demonstrably equivalent if and only if $T \vdash \zeta \leftrightarrow \xi$

2. If $\zeta \in \mathscr{S}_{\mathrm{Ar}}, \xi \in \mathscr{S}_{T}$, and $\zeta$ and $\xi$ are $T$-demonstrably equivalent, then we say that $\xi$ is arithmetically expressible within $T$.

We will write $\mathbf{N}$ for the standard model where the axioms of arithmetic are satisfied. If WF is the von Neumann well-founded set-theoretic universe 
(Manin, 1977), then $\mathbf{N} \subset \mathbf{W F}$; the same is true of the other standard settheoretic models, including Cohen's (1966) minimal constructive model.

However, we need a stronger condition on the semantics of $T$ :

Definition 2.4. If $L_{T}^{1} \supset L_{\mathrm{Ar}}^{1}$, then we say that $T$ is arithmetically consistent if and only if $\mathbf{N}$ is a model for all sentences in $\mathscr{S}_{\mathrm{Ar}}$ that are provable in $T$.

Remark 2.5. From here on we suppose that all theories we are handling are consistent in the particular sense made explicit above: for any theory $T$ such that $L_{T}^{1} \supset L_{\mathrm{Ar}}^{1}$, every arithmetic theorem of $T$ is true in the standard model N. That condition means that one of the models for arithmetic in $T$ is the standard model $\mathbf{N}$. That is the content of our definition of an arithmetically consistent theory.

\section{RICHARDSON'S THEOREM}

Several authors have dealt in recent times with the concept of computability (and with noncomputable objects and undecidable questions) in the realm of classical analysis. We can quote the Pour-El characterization for computability in function spaces and its application to mathematical physics (Pour-El and Caldwell, 1975; Pour-El and Richards, 1981, 1983a,b, 1989). More recently, Blum et al. (1989) have started a theory of computability over the reals, and have obtained nonrecursive recursively enumerable objects with respect to their point of view in the theory of dynamical systems.

However, we are going to use here a much older series of results, stemming from Richardson's (1968) paper on the undecidability of real analysis out of undecidable problems that arise in the theory of exponential Diophantine and plain Diophantine equations. Richardson's main lemma can be seen as the construction of a functor from the (small, i.e., set-theoretic) category of Diophantine equations into the algebra of elementary real-valued functions defined over $\mathrm{R}^{n}, n$ finite, and from that algebra into the algebra of elementary functions of a single real variable. Since the theory of Diophantine equations and of the associated Diophantine sets perfectly mirrors recursion theory on the natural numbers $\omega_{0}$ (Davis et al., 1976), we can reproduce with no great effort several standard results in recursion theory within both algebras of elementary functions, and that includes its undecidability features.

We note that undecidability results and a more systematic investigation of the incompleteness phenomenon in classical analysis with techniques that stem directly out of Gödel's original incompleteness theorem of 1931 are subjects very infrequently dealt with in the recent literature, as we can verify 
from an examination of the 1985 to beginning 1990 issues of the Mathematical Reviews. Results along the lines of those by Richardson have been independently obtained by Adler (1969) and Scarpellini (1963). Papers that stemmed from Richardson's are Caviness (1970), Richardson (1969), and Wang (1974); we have used portions of the Caviness-Wang reformulation of Richardson's results. However, we note that a more recent review of nonalgorithmicity (Davenport, 1981) quotes only Richardson's paper.

\subsection{The Main Ideas}

Real analysis deals with objects that are in general sets with the cardinality of the continuum. Therefore, in order to manipulate at least some of those sets, we represent them through expressions in the language $L_{T}^{1}$ of the theory $T$. Given an algebra of functions $\mathscr{D}$, we try to obtain a set of expressions $E(\mathscr{D})$ in $L_{T}^{1}$ for the functions in $\mathscr{D}$, so that given any $f \in \mathscr{D}$, there is at least one $E(f) \in E(\mathscr{D})$ that represents the function $f$. The idea is that $E(\mathscr{D})$ should be decidable in $L_{T}^{1}$ [that is, given a string $\xi \in L_{T}^{1}$, we must be able to check whether $\xi$ is in $E(\mathscr{D})$ or not].

For example, given the alphabet $\left\{x,+,-, \mid,(),,{ }^{\prime},\right\}$ we are able to represent through finite strings of symbols over that alphabet all polynomials of a real variable with rational coefficients. Convenient abbreviations allow us to handle rational polynomials with the usual notation.

We are going to consider an algebra of (at least sectionally smooth) real-defined and real-valued functions built out of elementary functions, that is to say, an algebra whose main "bricks" are polynomials, sines, cosines, base- $e$ exponentials, plus rational coefficients and the number $\pi$; we will extend that algebra to include the absolute value function $|x|$, the sign function $\eta(x)$, rational functions, some derivatives, and numbers defined by (proper or improper) definite integrals with limits expressible with finite strings of symbols. The corresponding set of expressions $E$ will, however, abide to the recursiveness property stated above, and expressions in $E$ will look like the usual objects that one handles in an elementary course in analysis, even if they will sometimes be very large and complicated expressions.

Now, do we have a $1-1$ correspondence between $\mathscr{D}$ and $E(\mathscr{D})$ ? No: both $f(x)=0$ and $g(x)=x \cos (\pi / 2)$ are expressions for the everywhere null function. Also, we will see below that we cannot in general algorithmically check whether two different expressions in $E(\mathscr{D})$ represent the same function in $\mathscr{D}$.

Given an expression in one of our $E(\mathscr{D})$, we can compute for an arbitrary computable set of values of the variables in the expression the value of the function represented by that expression, as every "brick" in $E(\mathscr{D})$ is 
a computable function in all standard concepts for the computability of real functions (Pour-El and Caldwell, 1975; Pour-El and Richards, 1981, $1983 a, b, 1989)$. Since we will only have real-defined and real-valued functions with a nice set of discontinuities, such functions will be defined when we know their values at the rationals. Therefore, it is easily seen that undecidable questions will appear as soon as we start the development of their theory. For example: given an arbitrary expression for a function in one of the $\mathscr{D}$ that appear in the present work, we cannot in general algorithmically decide whether that expression represents the zero function. Compute it: fix an enumeration for $Q$ and start checking value after value. If the expression is nonzero somewhere, the computation will eventually find that nonzero value, and stop. If it is always zero, the computation will never stop (J. Stolfi, personal communication). Richardson's functor allows us to obtain such undecidability results (and related incompleteness results) out of the more familiar undecidability and incompleteness results in formalized arithmetic.

Also, such algebras $\mathscr{D}$ are the main staple of theoretical physics. Therefore, once we have obtained undecidability and incompleteness results for them, we can reinterprete those results within physics.

\subsection{Polynomials and Subelementary Functions}

Let $P\left[x_{1}, x_{2}, \ldots, y_{1}, y_{2}, \ldots, Z\right]$ be the ring of polynomials in the (infinite denumerable) set of unknowns

$$
\left\{x_{1}, x_{2}, x_{3}, \ldots, y_{1}, y_{2}, y_{3}, \ldots\right\}
$$

with coefficients in the set $Z$ of integers, $P$ is closed under addition, multiplication, and function composition. $P$ is also a denumerable set.

Let $p\left(x_{1}, x_{2}, \ldots, x_{m}, y_{1}, y_{2}, \ldots, y_{n}\right) \in P$ be a polynomial in the $m+n$ unknowns $x_{1}, \ldots, x_{m}, y_{1}, \ldots, y_{n}$, with $m \geq 0$. Then we define:

Definition 3.1. The set $D$,

$$
\left\langle x_{1}, \ldots, x_{m}\right\rangle \in D \leftrightarrow \exists y_{1}, \ldots, y_{n} \in \omega_{0} p\left(x_{1}, \ldots, x_{m}, y_{1}, \ldots, y_{n}\right)=0
$$

where the $x_{i} \in \omega_{0}$, is a Diophantine set.

For completeness we add the corresponding results for exponential Diophantine equations: let us add to the elements of $P$ exponential expressions as in Davis et al. $(1961,1976)$; we get the ring $E\left[x_{1}, \ldots, Z\right]$ of exponential polynomials over $Z$. It is again a denumerable set. 
Let $e\left(x_{1}, \ldots, x_{m}, y_{1}, \ldots, y_{n}\right) \in E$. We define:

Definition 3.2. The set $D$,

$$
\left\langle x_{1}, \ldots, x_{m},\right\rangle \in D \leftrightarrow \exists y_{1}, \ldots \in \omega_{0} e\left(x_{1}, \ldots, x_{m}, y_{1}, \ldots, y_{n}\right)=0
$$

where the $x_{i} \in \omega_{0}$, is an exponential Diophantine set.

We have the two well-known results:

Proposition 3.3. 1. $D$ is exponential Diophantine if and only if $D$ is a recursively enumerable relation on $\omega_{0}^{m}$.

2. $D$ is Diophantine if and only if $D$ is a recursively enumerable relation on $\omega_{0}^{m}$.

Proof. For part 1, see Davis et al. (1961) and Jones and Matijaševič (1984); for part 2, see Davis (1973).

Corollary 3.4. Recursively enumerable relations on the positive integers are definable in the first-order language $L_{\mathrm{Ar}}^{1}$ of arithmetic.

Proof. See Manin (1977).

\subsection{Richardson's Functor}

Richardson's argument is developed (Richardson, 1968) within informal mathematics, but it can be adequately and rigorously formalized within ZFC, which we suppose to be consistent in the sense that all arithmetical theorems of ZFC are true in the standard model $\mathbf{N}$ for arithmetic; that is, our theories are, as we have already pointed out, arithmetically consistent. Richardson's main result is, for every arithmetic set of sentences (Davis, 1973; Davis et al., 1976; Rogers, 1967) $\left\{\varphi_{m}\right\}$, parametrized by a natural number $m \in \omega_{0}$, there is a set-theoretic set of sentences $\left\{\Phi_{m}\right\}$, again parametrized by the natural numbers, so that for every $m, \mathrm{ZFC} \vdash \varphi_{m} \leftrightarrow \Phi_{m}$. As an arithmetic predicate, $\varphi_{m}$ is an assertion about natural numbers, while $\Phi_{m}$ is a sentence involving elementary functions and real numbers.

Therefore $\left\{\Phi_{m}\right\}$ is a set of arithmetically expressible sentences within ZFC.

We will refer to this system of equivalences as "Richardson's functor," as it provides a way of translating statements about natural numbers into statements about real numbers, that is, we get an equivalence between sentences which are formalized in arithmetic, and sentences which are formalized in the theory of elementary functions of a real variable, a theory that includes arithmetic as one of its subtheories.

Richardson's results then arise from the fact that, for some sets of such sentences, $K=\{m: Z F C \vdash \varphi(\hat{m})\}$, where $\hat{m}$ is the name of $m$ within ZFC, 
is not a recursive set, which is equivalent to the nonrecursiveness of $K=$ $\{m: Z F C \vdash \Phi(\hat{m})\}$. Moreover, since we are dealing with arithmetically expressible sentences, the same nonrecursiveness of $K$ implies that there is a $k_{0} \in \omega_{0}-K$ such that $\mathbf{N} \vDash \neg \varphi\left(\hat{k}_{0}\right)$, but neither $\varphi\left(\hat{k}_{0}\right)$ nor its negation can be proved within arithmetic. Therefore, $\mathbf{M} \vDash \neg \Phi\left(\hat{k}_{0}\right)$, but neither $\Phi\left(\hat{k}_{0}\right)$ nor its negation can be proved within ZFC.

We will actually restrict our attention at the beginning to an algebra of subelementary functions; we will also handle the corresponding set of expressions that represent those functions in a convenient $L_{T}^{1}$. We define the algebra $\mathscr{A}\left[x_{1}, \ldots, 0\right]$ of subelementary functions in the unknowns $\left\{x_{1}, \ldots\right\}$ over the rational numbers $\mathrm{Q}$. It is thus constructed:

1. $\pi$ and $q \in \mathscr{A}$, where $q \in \mathrm{Q}$.

2. $x_{i}, \sin x_{i}$, and $e^{x_{i}} \in \mathscr{A}$.

3. If $A$ and $B \in \mathscr{A}, A+B$ and $A \cdot B \in \mathscr{A}$.

4. If $A$ and $B \in \mathscr{A}, A \circ B \in \mathscr{A}$ ( $\circ$ denotes function composition).

5. $\mathscr{A}$ is the smallest algebra closed under the preceding conditions.

Lemma 3.5. $\mathscr{A}$ is closed under partial derivation $\partial_{i}=\hat{\partial}_{z x_{i}}$.

Proof. Immediate.

Remark 3.6. $\mathscr{A}$ is a denumerable ring, since it has been defined over $\mathrm{O}$, but it is convenient to look at it as embedded into its real extension $\mathscr{A}[\mathrm{R}]$. However, we will not change our notation; whenever we need to go back to a denumerable ring, that fact will be explicitly noted.

From here on, if $\mathscr{D}$ is a set of functions, $E(\mathscr{D})$ is the set of those expressions in $L_{T}^{1}$ that represent the functions in $\mathscr{D}$. When we say that we can construct a function, we mean that we can construct the corresponding expression in $E(\mathscr{D})$. Also, functions in any set $\mathscr{D}$ are handled through the expressions in $E(\mathscr{D})$, so that, e.g., decision problems for $\mathscr{D}$ are in fact decision problems for $E(\mathscr{D})$.

Lemma 3.7. If $f \in \mathscr{A}$, then there is $g \in \mathscr{A}$ so that:

1. $\forall x_{1} \cdots x_{n} \in \mathrm{R} g\left(x_{1}, \ldots, x_{n}\right)>1$

2. $\forall x_{1} \cdots x_{n} \in \mathrm{R}, \Delta_{1}, \ldots, \Delta_{n} \in \mathrm{R},\left(\left|\Delta_{1}\right| \leq 1 \wedge \cdots \wedge\left|\Delta_{n}\right| \leq 1\right)$

$\rightarrow\left(g\left(x_{1}, \ldots, x_{n}\right)>\left|f\left(x_{1}+\Delta_{1}, \ldots, x_{n}+\Delta_{n}\right)\right|\right)$

Proof. Proof is by induction. First step: we deal with constants or variables.

(i) If

$$
f\left(x_{1}, \ldots, x_{n}\right)=c
$$


$c$ a constant, then

$$
g\left(x_{1}, \ldots, x_{n}\right)=|c|+2
$$

(ii) If

$$
f\left(x_{1}, \ldots, x_{n}\right)=x_{i}
$$

then

$$
g\left(x_{1}, \ldots, x_{n}\right)=x_{i}^{2}+2
$$

Clearly, (1) is satisfied, as always $g>1$. For (2), we have

$$
x_{i}^{2}+2>\left|x_{i}+\Delta_{i}\right|
$$

$\left|\Delta_{i}\right| \leq 1$. For $x^{2}>x, x>1$, and the constant 2 takes care of the situation for $0<x \leq 1$.

Induction step: suppose that $A\left(x_{1}, \ldots, x_{n}\right)$ and $B\left(x_{1}, \ldots, x_{n}\right)$ are obtained in $k$ or less steps. Suppose also that we have constructed $g$ and $h$ such that

$$
\begin{aligned}
& g\left(x_{1}, \ldots, x_{n}\right)>\left|A\left(x_{1}+\Delta_{1}, \ldots, x_{n}+\Delta_{n}\right)\right| \\
& g\left(x_{1}, \ldots, x_{n}\right)>1
\end{aligned}
$$

Similar conditions $[h(x)>1$ and $h(\boldsymbol{x})>|B(\boldsymbol{x}+\boldsymbol{\Delta})|$, where we abbreviate $\boldsymbol{x}=$ $\left.\left\langle x_{1}, \ldots, x_{n}\right\rangle\right]$ are valid of $h$. Then:

- If $f=A \pm B$, we put $k=g+h$.

- If $f=A B$, we put $k=g h$.

- If $f=e^{A}$, we put $k=e^{g}$.

- If $f=\sin A$, we put $k=2$.

We thus conclude the proof.

We can now state the first of Richardson's main results: given that $L_{\mathrm{Ar}}^{1} \subset L_{\mathrm{ZFC}}^{1}$ :

Proposition 3.8 (Richardson's Functor, I). There is an injection $\imath_{P}: P \rightarrow \mathscr{A}$, where $P$ denotes the algebra of Z-valued polynomials in a finite number of variables, and $\mathscr{A}$ is the algebra of subelementary functions described above, such that:

1. $l_{P}$ is constructive, that is, given the expression for $p$ in $L_{\mathrm{Ar}}^{1}$, there is an effective procedure so that we can obtain the corresponding expression for $F=\iota_{p}(p)$ in $L_{\mathrm{ZFC}}^{1}$.

2. $t_{P}$ is $1-1$.

3. $\exists \boldsymbol{x} \in \omega_{0}^{n} p(m, \boldsymbol{x})=0$ if and only if $\exists \boldsymbol{x} \in \mathrm{R}^{n} F(m, \boldsymbol{x})=0$ if and only if $\exists x \in R^{n} F(m, x) \leq 1$, for $p \in P$ and $F \in \mathscr{A}$. 
4. The injection $l_{P}$ is proper.

Proof. For the first statement, proof follows from the following lemma and constructive definitions for $\imath_{P}(p)$ :

Lemma 3.9. There is a constructive procedure such that given the expression for $p \in P$, we can obtain the expressions for functions $k_{i} \in \mathscr{A}$ that satisfy the following conditions: if $\left|\Delta_{i}\right| \leq 1$, real numbers, then

$$
k_{i}\left(m, x_{1}, \ldots, x_{n}\right)>\left|\partial_{i}\left(p^{2}\left(m, x_{1}+\Delta_{1}, \ldots, x_{n}+\Delta_{n}\right)\right)\right|
$$

Proof. Apply the construction in Lemma 3.7 to $p$. We notice that the computation of the expressions for any such $\partial_{i} A$ is clearly algorithmic for subelementary functions explicitly given.

Definition 3.10. Given $p \in P$, for $k_{i}$ as in Lemma 3.9, we define

$$
\begin{aligned}
f\left(m, x_{1}, \ldots, x_{n}\right)= & (n+1)^{4}=(n+1)^{4}\left\{p^{2}\left(m, x_{1}, \ldots, x_{n}\right)\right. \\
& \left.+\sum_{i=1}^{n}\left(\sin ^{2} \pi x_{i}\right) k_{i}^{4}\left(m, x_{1}, \ldots, x_{n}\right)\right\}
\end{aligned}
$$

Finally, we write:

Definition 3.11. $F\left(m, x_{1}, \ldots, x_{n}\right)=f\left(m, x_{1}^{2}, \ldots, x_{n}^{2}\right)$.

We settle the first question.

For the second assertion, it is immediate that $p_{1} \neq p_{2}$ implies that $F_{1} \neq F_{2}$.

For the fourth assertion, we notice (Lemma 3.9) that it suffices to obtain a subelementary function as in Definition 3.10 , but such that $k<\left|\partial_{i} p\right|$. The resulting function is subelementary if $k$ is a subelementary function, but it cannot belong to the image of $t_{P}$.

Finally, the third assertion is the main content of Richardson's result, and is proved in (Richardson, 1968) in a straightforward way.

Remark 3.12. We define

$$
\begin{aligned}
& h(x)=x \sin x \\
& g(x)=x \sin x^{3}
\end{aligned}
$$

given $F\left(m, x_{1}, \ldots, x_{n}\right)$, we make the following substitutions:

$$
\begin{aligned}
x_{1} & =h(x) \\
x_{2} & =h \circ g(x) \\
x_{3} & =h \circ g \circ g(x) \\
& \vdots \\
x_{n-1} & =h \circ g \circ \cdots \circ g(x)
\end{aligned}
$$


(where $g$ is composed $n-2$ times), and

$$
x_{n}=g \circ g \circ \cdots \circ g(x)
$$

where here $g$ is composed $n$ times.

We thus get $G(m, x)$, defined over $\mathrm{R}$ and with values in $\mathrm{R}$, where as usual $m \in \omega_{0}$.

Corollary 3.13 (Richardson's Functor, II). Let $\mathscr{A}_{1}$ be the algebra of subelementary functions over a single real variable $x$. Then there is a map $\imath^{\prime}: P \rightarrow \mathscr{A}_{1}$ such that:

1. $t^{\prime}$ is constructive.

2. $t^{\prime}$ is $1-1$.

3. The inclusion $t^{\prime}(P) \supset \mathscr{A}_{1}$ is proper.

4. $\exists x \in \omega_{0}^{n} p(m, x)=0$ if and only if $\exists x \in R \quad G(m, x)=0$ if and only if $\exists x \in R \quad G(m, x) \leq 1$.

Proof. $G(m, x)$ is given in Remark 3.12. Given $F$, the corresponding expression for $G$ is obviously constructive. We therefore prove the first assertion.

The second assertion is again a consequence of the fact that given two different polynomials over $Q, p_{1} \neq p_{2}$ entails that $G_{1} \neq G_{2}$. The third assertion is also proved as in the preceding theorem. Finally, for the last assertion, see the proof in Richardson (1968).

The next two results take place in the algebra $\mathscr{A}_{1}^{\prime}$, which is $\mathscr{A}_{1}$ with the new function $\sqrt{x}$ and closed under this new operation and the previous ones.

Definition 3.14. We write:

1. $|x|=+\sqrt{x^{2}}$

2. $x^{*} y=(1 / 2)(|x-y|+(x-y))$

3. $\min (x, y)=x-\left(x^{*} y\right)$

Corollary 3.15. If

$$
B(m, x)=|G(m, x)-1|-(G(m, x)-1)
$$

then $\forall x \in \mathbf{R} B(m, x)=0$ if and only if $\neg \exists x \in \mathbf{R} G(m, x)<1$.

Proof. Immediate, from the definition of $B$.

Corollary 3.16. If

$$
\chi(m, x)=\min (1,2 * 2 G(m, x))
$$


then:

1. $\neg \exists x \in \mathbf{R} G(m, x)<1$ if and only if $\forall x \in \mathbf{R} \chi(m, x)=0$.

2. $\exists x \in \mathrm{R} G(m, x)<1$ if and only if there is an interval $I_{m} \subset \mathrm{R}$ such that we have $\forall x \in I_{m} \chi(m, x)=1$.

Proof. See Richardson (1968). for $m$.

We wish to emphasize that each $I_{m}$ depends on the particular value

Caviness (1970) and Wang (1974) have improvements on the preceding results.

Remark 3.17. From now on we suppose that we have extended $\mathscr{A}_{1}^{\prime}$ to an algebra 2 that includes rational functions $f / g, g, g \in \mathscr{A}_{1}^{\prime}$, plus real numbers defined by (proper or improper) definite integrals of the form $\int_{a}^{b} f(x) d x$, where again $f \in \mathscr{A}_{1}^{\prime}$. We also add to $\mathscr{Q}$ the step function $\eta(x)$,

$$
\begin{array}{ll}
\eta(x)=x /|x|, & x \neq 0 \\
\eta(x)=0, & x=0
\end{array}
$$

If we understand that derivations in 2 include derivations of continuous functions in the sense of distribution theory (Jantscher, 1971, pp. 86 and 319 ; and Yosida, 1980), we will obtain in $E(2)$ and expression for $\eta$ involving only the derivative and the absolute value function $|\cdots|$ :

$$
\eta(x)=\frac{d}{d x}|x|
$$

We take 2 to be closed under (finite) algebraic operations and function composition.

We state:

Corollary 3.18. There is a function $\theta(m, x) \in \mathscr{Q}$ so that:

1. $\neg \exists x \in \mathbf{R} G(m, x)<1$ if and only if $\forall x \in \mathbf{R} \theta(m, x)=0$.

2. $\exists x \in \mathbf{R} G(m, x)<1$ if and only if $\forall x \in \mathbf{R} \theta(m, x)=1$.

Proof. We can actually construct an expression for $\theta(m, x)$ in 2. Put $C(m, x)=(B(m, x))^{2}$, for $B$ as in Corollary 3.15. Then, for every real $x$, $C(m, x) \geq 0$. The succession of definite integrals

$$
K(m)=\int_{-\infty}^{+\infty} \frac{C(m, x) e^{-x^{2}}}{1+C(m, x)} d x
$$


is convergent, for all values of $m$. We can thus write

$$
\theta(m, x)=\theta(m)=\eta\left(\frac{K_{m}}{1+K_{m}}\right)
$$

Remark 3.19. If what we really want to obtain out of Richardson's results is a simple, convenient, two-state, smooth, real function that distinguishes between the two possibilities embodied in $G(m, x)$ in Corollary 3.13, we can proceed according to the following suggestion by J. Stolfi (personal communication): $G(m, x)$ behaves as follows-either we have $\forall x \in \mathrm{R} G(m, x) \geq 1$ or $\exists x \in \mathrm{R} G(m, x)<1$.

We can therefore directly define $\theta(m): \theta(m)=0$ if and only if $G(m, x) \geq 1$, always, and $\theta(m)=1$ if and only if $G(m, x) \leq(1 / 2)$ somewhere.

However, even if that construction is quite natural, we would like to obtain $\theta(m)$ as an expression involving elementary functions plus "nice" operations such as the absolute value or the derivative, as we did above.

Moreover, $\theta(\mathrm{m})$ does not have a unique expression in the algebra $\mathscr{Q}$, as the unsolvability of the identity problem (see Corollary 3.15 above and Corollary 3.22 below) for elementary functions with an absolute value function added allows for the possibility that many other expressions within $\mathscr{2}$ might represent $\theta(m)$, without that fact being algorithmically verifiable, since we cannot algorithmically decide in the general case whether $\theta(m, x) \equiv f(m, x)$, for an arbitrary $f(m, x)$ within $\mathscr{Q}$.

It is also worth emphasizing that $\theta(m, x)$ is a smooth family of functions, even if defined out of the step function $\eta$.

Another possibility for a two-state function like $\theta$ is given in da Costa and Doria (1990b). Of course, the main motivation for the construction of one such $\theta(m)$ is that, if we start from a universal polynomial $p(m, x)$ (Jones, $1982)$, the corresponding $\theta(m)$ will represent, with the help of elementary functions and operations in real analysis, a function that "solves" the Halting Problem for Turing machines (Jones, 1982; Rogers, 1967; Scarpellini, 1963). There are assuredly simpler constructions than the one we have given above; the algorithmic undecidability of the identity problem for elementary functions (see Corollary 3.22) also ensures that there is an infinity of such $\theta(m)$ in 2 , which we may never algorithmically recognize as "representations" for the Halting Problem.

\subsection{Undecidability and Incompleteness}

We note that all the preceding maps and equivalences can be adequately formalized as ZFC theorems. 
Our main undecidability results stem from the following:

Lemma 3.20. 1. There is a Diophantine set $D$ so that

$$
m \in D \leftrightarrow \exists x_{1}, \ldots, x_{n} \in \omega_{0} p\left(m, x_{1}, \ldots, x_{n}\right)=0
$$

$p \in P$, and $D$ is recursively enumerable but not recursive.

2. There is an exponential Diophantine set $D$ so that

$$
m \in D \leftrightarrow \exists x_{1}, \ldots, x_{n} \in \omega_{0} e\left(m, x_{1}, \ldots, x_{n}\right)=0
$$

$e \in E$, and $D$ is recursively enumerable but not recursive.

Proof. For part 1, see Davis (1973) and Manin (1977). For part 2 see Davis et al. (1961) and Jones and Matijaševič (1984).

Corollary 3.21. For an arbitrary $m \in \omega_{0}$ there is no general decision procedure to check whether $p\left(m, x_{1}, \ldots\right)=0$ or $e\left(m, x_{1}, \ldots\right)=0$ have a solution in the positive integers.

We will not explore the results associated to exponential Diophantine equations, since we can restrict our attention to the Diophantine situation. However, from time to time we will mention the possible extension to the exponential Diophantine case.

We will now handle functions in the algebra 2 that are Richardson transforms of a $p$ that satisfies Corollary 3.21. We will consider such a polynomial $p$ that a given arithmetical sentence $\psi(p)$ is undecidable in formalized arithmetic, and will get through Richardson's functor several sentences $\Psi\left(\imath^{\prime}(p)\right)$ that are demonstrably equivalent to $\psi(p)$ in ZFC; the equivalence implies that they are also undecidable in ZFC, which we suppose to be arithmetically consistent. See Proposition 3.28. Similar constructions can also be made for the corresponding exponential Diophantine expression $e$.

That means, for $p$ as above,

1. For $m \in \omega_{0}$, there is no general algorithm to decide whether there are natural numbers $x_{1}, \ldots$ so that $p\left(m, x_{1}, \ldots\right)=0$.

2. $p: \omega_{0}^{n+1} \rightarrow \mathrm{Z}$.

Therefore, given such a $p$, and $F=\imath_{P}(p)$, and the other related functions ( $G, B$, and so on), we have:

Corollary 3.22. For an arbitrary $m \in \omega_{0}$ there is no general procedure to check whether:

1. There are real numbers $x_{1}, \ldots, x_{n}$ such that $F\left(m, x_{1}, \ldots, x_{n}\right)=0$.

2. There is a real number $x$ so that $G(m, x)<1$.

3. Whether we have $\forall x \in R B(m, x)=0$, for $B$ as in Corollary 3.15. 
4. Whether we have $\forall x \in \mathbf{R} \chi(m, x)=0$ or $\forall x \in I \quad \chi(m, x)=1$ over an interval $I_{m}$, as in Corollary 3.16.

5. Whether we have $\forall x \in \mathbf{R} \quad \theta(m, x)=0$ or $\forall x \in \mathbf{R} \quad \theta(m, x)=1$ over the reals, as in Corollary 3.18 .

6. Whether for an arbitrary $f(m, x)$ we have $f(m, x) \equiv \theta(m, x)$.

Proof. From the preceding results. The last undecidability statement follows from the third one.

Remark 3.23. We can go further: for example, as the embedding $\imath_{P}(P) \subset \mathscr{A}_{1}^{\prime}$ is proper, is there a general decision procedure to check whether a given $f \in \mathscr{A}_{1}^{\prime}$ is the image of some $p \in P$ ? The answer is, no. Let $B(m, x)$ be as in Corollary 3.15, and let $c_{0}, r \in \mathscr{A}_{1}^{\prime}-l_{P}(P)$. Then, for

$$
f(m, x)=c_{0}+B(m, x) r(m, x)
$$

there is no such a decision procedure.

The present example is a kind of blueprint for a family of undecidability results in $\mathscr{A}_{1}^{\prime}$ and its extensions. See Proposition 3.27 below.

Therefore, lists of functions such as $G(m, x), B(m, x)$, and the like have several undecidability properties, that is to say, we cannot construct a general algorithm that, for each $m$, allows us to decide propeties like those described above.

However, there is more in stock here: we now quote a result that comes straight down from a famous paper by Post (1944). Post gave in that paper an intuitive proof of a Gödel-like incompleteness theorem in arithmetic, and that proof was imitated in the proof of an incompleteness theorem for exponential Diophantine equations in Davis et al. (1961) and later in a similar result for plain Diophantine equations (Davis, 1973; Davis et al., 1973).

Let $T$ be an arithmetically consistent first-order theory whose language $L_{T} \supset L_{\mathrm{Ar}}^{1}$. Suppose that the set of axioms of $T$ is recursively enumerable. Suppose also that one can prove form $T$ every statement of the form $m+n=$ $p, m \cdot n=p$ and $m<n$, where $m, n \in \omega_{0}$, which is true in the standard model N. Then:

Proposition 3.24. We can construct in $T$ a Diophantine equation

$$
p\left(x_{1}, \ldots, x_{n}\right)=0
$$


so that $p=0$ has no solutions in the natural numbers in $\mathbf{N}$, but such that

$$
T \nvdash \neg\left(\exists x \in \omega_{0}^{n} p(\boldsymbol{x})=0\right)
$$

Proof. See Davis (1973) and Davis et al. (1976). Notice that we cannot also prove

$$
T \vdash\left(\exists x \in \omega_{0}^{n} p(x)=0\right)
$$

since in that case $\mathbf{N}$ would not be a model for arithmetic within $T$, as $T$ is supposed to be arithmetically consistent. Therefore, the sentence

$$
\exists x \in \omega_{0}^{n} p(x)=0
$$

is undecidable in $T$, for this particular $p$.

Corollary 3.25. If $\mathrm{ZFC}$ is arithmetically consistent, then neither $\mathrm{ZFC} \nvdash \exists \boldsymbol{x} \in \omega_{0}^{n} p(\boldsymbol{x})=0$ nor $\mathrm{ZFC} \nvdash \neg \neg\left(\exists \boldsymbol{x} \in \omega_{0}^{n} p(\boldsymbol{x})=0\right)$. Moreover, for a model $\mathbf{M}$ of ZFC such that $\mathbf{N} \subset \mathbf{M}$ is a model for arithmetic in ZFC, then $\mathbf{M} \vDash \neg\left(\exists x \in \omega_{0}^{n} p(x)=0\right.$.

We now get the Richardson ZFC-demonstrable equivalence:

Lemma 3.26.

$$
\mathrm{ZFC} \vdash \neg\left(\exists x \in \omega_{0}^{n} p(x)=0\right) \leftrightarrow \neg \exists x \in \mathrm{R}^{n} F(x) \leq 1
$$

Proof. Above.

Corollary 3.27. If ZFC is arithmetically consistent, then neither $\mathrm{ZFC} \nvdash \xi$, nor $\mathrm{ZFC} \nvdash \neg \xi$, but $\mathbf{M} \vDash \xi$, for $\xi$ as follows:

1. $\neg\left(\exists x \in \mathrm{R}^{n}\right) F(x) \leq 1$.

2. $\neg\left(\exists x \in R^{n}\right) F(x)=0$.

3. $\forall x \in \mathrm{R} B(x)=0$.

4. For $\theta$ as in Corollary 3.18, $\forall x \in \mathrm{R} \theta(x)=0$.

5. There is an $f(m, x)$ whose expression does not coincide with the expression for $\theta(m, x)$ in Corollary 3.18 , but such that $\forall x \in \mathbf{R}$ $f(m, x)=\theta(m, x)$.

Proof. From the preceding propositions. For the last statement we write $f(m, x)=B(x)+\theta(m, x)$, for $B(x)$ as above in 3.

There is a general undecidability and incompleteness result at work here (P. Suppes, private comment). Everything proceeds within ZFC (or within any similarly powerful axiomatic system $T$ ), so that we can obtain all the 
maps given by Richardson's functor into $\mathscr{A}$ and extensions. Let also $\mathscr{B} \subseteq \mathscr{2}$ be any superalgebra; let $\psi \in L_{\mathrm{ZFC}}^{1}$ be a predicate defined for $\mathscr{B}$ such that

$$
\mathrm{ZFC} \vdash \exists f \in \mathscr{B} \exists g \in \mathscr{B} \psi(f) \wedge \neg \psi(g)
$$

If $\xi \in L_{\mathrm{ZFC}}^{1}$ is any expression in that language, we write $\|\xi\|$ for its complexity, as measured by the number of letters from ZFC's alphabet in $\xi$. Also we define the complexity of a proof $C_{\mathrm{ZFC}}(\xi)$ of $\xi$ in $L_{\mathrm{ZFC}}^{1}$ to be the minimum length that a deduction of $\xi$ from the ZFC axioms can have, as measured by the total number of letters in the expressions that belong to the proof.

Then:

Proposition 3.28. If ZFC is arithmetically consistent, then:

1. There is an $h \in \mathscr{B}$ so that neither $\mathrm{ZFC} \nvdash \neg \psi(h)$ nor $\mathrm{ZFC} \nvdash \psi(h)$, but $\mathbf{M} \vDash \psi(h)$, where $\mathbf{M}$ is as in Corollary 3.25.

2. There is a denumerable set of functions $h_{m}(x) \in \mathscr{B}, m \in \omega_{0}$, such that there is no general decision procedure to ascertain, for an arbitrary $m$, whether $\psi\left(h_{m}\right)$ or $\neg \psi\left(h_{m}\right)$ is provable in ZFC.

3. Given the set $K=\{m: \mathrm{ZFC}-\psi(\hat{m})\}$, and given an arbitrary total recursive function $g: \omega_{0} \rightarrow \omega_{0}$, there is an infinite number of values for $m$ so that $C_{\mathrm{ZFC}}(\psi(\hat{m}))>g(\|\psi(\hat{m})\|)$.

Proof. Let $\theta$ be as in Corollary 3.18. Let $f_{0}, g_{0}$ satisfy our conditions on $\psi$, that is, $\mathrm{ZFC} \vdash \psi\left(f_{0}\right)$ and $\mathrm{ZFC} \vdash \neg \psi\left(\mathrm{g}_{0}\right)$. Then define

$$
h(m, x)=\theta(m, x) f_{0}+[1-\theta(m, x)] g_{0}
$$

This settles part 2. Now let us specify $\theta$ as in Corollary 3.27 , so that the corresponding Diophantine equation $p=0$ is never solvable, while that fact cannot be proved in ZFC. We then form, for such an indicator function,

$$
h=\theta f_{0}+(1-\theta) g_{0}
$$

This settles part 1 .

Finally, for part 3, we notice that as $K$ is recursively enumerable but not recursive, it satisfies the conditions in the Gödel-Ehrenfeucht-Mycielski theorem (Ehrenfeucht and Mycielski, 1971; Manin, 1977).

Remark 3.29. Notice that if we restrict $\mathscr{B}$ to its smooth subalgebra $\mathscr{B}^{s}$, which includes the halting function $\theta(m, x), \mathscr{B}^{s}$ can be included in anyone of the algebras or function spaces that commonly appear in theoretical physics. Examples are the Fréchet spaces $C^{k}(U, \mathrm{R})$, where $0 \leq k \leq+\infty$, of functions defined on an open domain $U \subseteq \mathrm{R}^{n}$, or its extensions; the Clifford modules $\mathscr{B}^{s} \otimes \mathbf{C}(Q)$, where $\mathbf{C}(Q)$ is the Clifford algebra over the quadratic form $Q$; the $C^{*}$-algebras $\mathscr{B}^{s} \otimes C$, where $\mathscr{B}^{s}$ is a commutative $C^{*}$-algebra that includes our extended algebra of adequately smooth subelementary 
functions, and $C$ is an arbitrary $C^{*}$-algebra, and so on. Smooth section spaces [spaces of classical physical fields (da Costa and Doria, 1990a)] also easily fit into the picture, as well as several spaces of Lebesgue-integrable functions.

The third result in Proposition 3.28 means that even if we can hope to prove that some objects do have the property $\psi$, the proofs for that fact can be inordinately long, that is to say, they will be "very hard" within the usual ZFC axioms. The other portion of Gödel's 1936 theorem, in the Ehrenfeucht-Mycielski formulation, however, suggests that if we add to ZFC the "right" axioms, the proofs for some intractable properties might be considerably shortened. The situation might be compared to what happens in analytical number theory or in algebraic geometry, when a very strong and elaborate mathematical machinery is called into action to solve problems in number theory.

But that step might also take us in some cases beyond the pale of standard mathematics, which is here represented by ZFC, into an assuredly controversial area.

\section{UNDECIDABILITY IN CLASSICAL MECHANICS}

We formalize classical mechanics with the tools described in da Costa and Doria (1990a). Everything therefore is supposed to be conveniently obtained as a Suppes predicate within ZFC. From now on when we say that ZFC is arithmetically consistent, we mean that ZFC has a model $\mathbf{M}$ whose arithmetical submodel $\mathbf{N}$ is standard.

\subsection{Motions}

Our first proposition gives an idea of the kind of result we can get out of Richardson's functor. It is interesting in itself, since it defies intuition, and also due to the fact that it leads to a direct albeit informal proof of one of our Gödel-like incompleteness results (see Proposition 5.3) whose main line of argument goes back to Post (1944).

A motion in $\mathbf{R}^{n}$ is a smooth map $m: \mathbf{R} \rightarrow \mathbf{R}^{n}$; in local coordinates it is given by the $n$-uple of smooth functions $m(t)=\left\langle m_{1}(t), \ldots, m_{n}(t)\right\rangle$.

Proposition 4.1. If ZFC is arithmetically consistent, then:

1. There is no general algorithmic procedure to determine, for any fixed open square $\mathrm{Sq} \subset \mathrm{R}^{2}$, whether an arbitrary motion

$$
m(t)=\left\langle m_{1}(t), m_{2}(t)\right\rangle
$$

will go through Sq. 
2. There is a motion $m$ on the plane $\mathrm{R}^{2}$ such that $\mathbf{M} \vDash m(t) \cap \mathrm{Sq}=\varnothing$, but $\mathrm{ZFC} \nvdash m(t) \cap \mathrm{Sq}=\varnothing$.

Remark 4.2. We cannot prove within our axiomatization that the motion does not go through the square, no matter how small it is. We emphasize that $m$ can be taken to be a smooth function, since we are here strictly working within the algebra $\mathscr{A}_{1}$ of subelementary functions of a single real variable.

Proof. For the first assertion: from Corollary 3.22, we define

$$
A(k, x)=G(k, x)-1
$$

Therefore, there is no general decision procedure to check whether one has, given an arbitrary $k \in \omega_{0}, A(k, x)<0$. Now make a coordinate change so that the upper boundary of the square coincides with the $x_{1}$ axis. Then put $m_{1}(k, t)=A(k, t)$. We are done.

For the second assertion: let $p$ be as in Proposition 3.24. Therefore, " $p=0$ has no solutions in $\omega_{0}$ " is undecidable in ZFC. As that assertion is equivalent to " $\forall x \in \mathbf{R} A(x) \geq 0$ " in $Z F C$, this new sentence is again undecidable in ZFC, where $A$ is built out of $p$ according to a recipe similar to those in the preceding section. As a result, given the motion $m$ so that $m_{1}(t)=$ $A(t)$, the sentence " $m(t) \cap \mathrm{Sq}=\varnothing$ " is undecidable in ZFC, even if there is a model $\mathbf{M}$ for $\mathrm{ZFC}$ where $\mathbf{M} \vDash m(t) \cap \mathrm{Sq}=\varnothing$.

\subsection{Hamiltonian Mechanics: The Integration Problem}

We take our motto from a quite well-known problem in classical mechanics. It is easy to write down a Lagrangian or a Hamiltonian for a given physical system, and to obtain the corresponding differential equations for the motion. We also know that if we are lucky enough to find or guess an adequate canonical transformation, we trivialize the system by mapping it onto a constant flow in phase space. The canonical transformation we need is the solution of the Hamilton-Jacobi equation. Is there a general algorithmic recipe to solve it? Numerical integration might be one such recipe, but it does not work, since we are in general dealing with quite complicated nonlinear systems that are highly unstable with respect to small changes in the initial conditions; they will very frequently have a "chaotic" behavior, and we may not be sure of the extent truncation errors will affect our results.

We therefore look for an algorithmic recipe that would work as follows: we are given an arbitrary Hamilton-Jacobi equation. Can we algorithmically check whether it is integrable by quadratures? Local integrals always exist, due to the Tubular Neighborhood theorem. But can we compute them? 
This problem has been around since the 19th century: it was recently discussed and evaluated in two textbooks that deal with chaos and integrability in dynamical systems. We first quote Tabor $(1989$, p. 322$)$ :

\begin{abstract}
A recurrent theme in the preceding chapters has been the distinction between integrable and nonintegrable systems. The latter can exhibit chaotic behavior, whereas the former are distinguished by the existence of a full complement of integrals and exhibit stable, multiply-periodic behavior.... Despite all the advances in nonlinear dynamics, a fundamental question still remains, namely: given a system of equations, how can one tell a priori whether or not they are integrable?
\end{abstract}

Tabor then discusses a few particular cases where there are methods for the determination of first integrals in Hamiltonian systems.

A similar discussion is also found in the following paragraph from Lichtenberg and Lieberman (1983, p. 38):

Are there any general methods to test for the integrability of a given Hamiltonian? The answer, for the moment is no. We can turn the question around, however, and ask if methods can be found to construct potentials that give rise to integrable Hamiltonians. The answer here is that a method exists, at least for a restricted class of problems, but the method becomes rapidly very tedious as the forms allowed for the integrals of the motion are expanded.

(Emphasis added to both quotations). Both Tabor, and Lichtenberg and Lieberman, assert here two things: first, the problem of finding a general integrability criterion for arbitrary Hamiltonian systems looks very difficult. We show below that it is, in fact, algorithmically unsolvable; there is no general algorithmic procedure to separate the quadrature-integrable Hamiltonians from those that are not quadrature-integrable.

Then they suggest that we try instead to experiment with particular sets of functions in order to check which ones might give rise to integrable Hamiltonian problems.

Here comes the main surprise: the obvious technique to develop integrable Hamiltonian systems would be to find a denumerable family of functions and test them for suitability as first integrals in Hamiltonian systems. We show that such a procedure also leads in the general case to an algorithmic impossibility.

To summarize:

1. Given a Hamiltonian $h$, do we have an algorithm that tells us whether the associated Hamilton dynamical system $X_{h}$ can be integrated by quadratures?

2. Given a Hamiltonian $h$ such that $X_{h}$ can be integrated by quadratures, can we algorithmically find a canonical transformation that will do the trick? 
3. Can we algorithmically check whether an arbitrary set of functions is a set of first integrals for a Hamiltonian system?

The answer to those questions is, no.

The mathematical setting for our results can be found in Arnold (1976) and da Costa and Doria (1990a). It goes as follows: let $P$ be a phase space, that is, a $2 n$-dimensional smooth real manifold endowed with a nondegenerate symplectic form $\eta$. A vectorfield $X$ on $P$ is (locally) Hamiltonian if $L_{X} \eta=$ 0 , where $L_{X}$ is the Lie derivative with respect to $X$. A function $f: U \subset P \rightarrow \mathrm{R}$ is a first integral of $X$ if $L_{X} f=0$. As $L_{X} \eta=0$ is locally equivalent (over a Poincare star-shaped domain) to $i_{X} \eta=-d h$, where $i_{X}$ is the left interior product with respect to $X, d$ is the exterior derivative and $h$ is the (local) Hamiltonian, we can show that (locally at least) $L_{X} f=0$ if and only if $\{f, h\}=0$, where $\{-,-\}$ denotes the Poisson bracket operation.

We also obtain the algebra $\mathscr{A}(U)$ of elementary functions over an open star-shaped domain $U \subset P$, as well any of its extensions $\mathscr{B}(U) \supset \mathscr{A}(U)$ as in Remark 3.29.

We will first consider the last question in the preceding list: Can we algorithmically check whether an arbitrary set of functions defined on phase space is a set of first integrals for a Hamiltonian system?

We say that a set $F=\left\langle f_{1}, \ldots, f_{k}\right\rangle, k \leq n$, of smooth functions on an open domain $U$ in a phase space $P$ of dimension $2 n$ is an independent set if and only if $\left\{f_{i}, f_{j}\right\}=0$, for all $i, j \leq k$. Then:

Proposition 4.3. Let us be given an arbitrary set of functions

$$
\Phi=\left\langle f_{1}, \ldots, f_{n}\right\rangle
$$

defined on a domain $U$ in phase space $P$. Then there is no general algorithmic procedure that allows us to determine whether $\Phi$ is an independent set on $U$.

Proof. We must show that the Poisson brackets $\left\{f_{i}, f_{i}\right\}=0$. Let $B\left(k, p_{1}\right)$ be the function $B$ in Proposition 3.22 with the obvious change of variables, and $k \in \omega_{0}$. Put

$$
f_{1}\left(k, q_{1}, p_{1}\right)=B\left(k, p_{1}\right) q_{1}
$$

Now write

$$
f_{2}=h=c p_{1}
$$

for $c \in \mathrm{R}$, a nonzero constant. Then,

$$
\left\{f_{1}, h\right\}=0 \leftrightarrow B\left(k, p_{1}\right)=0
$$

Now, as there is no decision procedure to check whether given an arbitrary $k \in \omega_{0}, \forall x \in \mathrm{R} B(k, x)=0$ or $B(k, x) \neq 0$, the independence of the set that 
includes the functions $f_{1}(k, q, p)$ as above cannot be algorithmically decided.

Notice that $h$ can be seen as the Hamiltonian for a particle whose trajectory in configuration space (restricted to $U$ ) is given by

$$
\left\langle c t, q_{02}, \ldots, q_{0 n}\right\rangle
$$

all $q_{0 i}$ kept constant.

Corollary 4.4. There is no algorithm to check, for an arbitrary Hamiltonian $h$ and for a smooth function $f$ defined on $P$, whether $f$ is a first integral of $h$.

Proof. Consequence of the preceding result.

We thus settle question 3 . We can now turn to the restricted version of the integrability problem: Given a Hamiltonian whose associated dynamical system has been proved to be integrable by quadratures, can we algorithmically find a canonical transformation that will allow us to explicitly integrate it?

Proposition 4.5. For an arbitrary Hamiltonian $h$ such that $X_{h}$ can be integrated by quadratures, there is no algorithm to solve the associated Hamilton-Jacobi equation.

Proof. We have two proofs; the first one goes as follows: we can use the "yes or no" function $\theta$, described in Corollary 3.18. We define the countable family of Hamiltonians

$$
h_{k}(q, p)=\frac{1}{2}\left(p^{2}+q^{2}\right) \theta(k, q)+p(1-\theta(k, q))
$$

For each $k \in \omega_{0}, h_{k}$ will either describe a free particle or a harmonic oscillator.

Therefore, given any $k, h_{k}$ is always integrable by quadratures.

Suppose then that there is an algorithm for solving the associated Hamilton-Jacobi equations. We could therefore decide whether each $h_{k}$ represents a harmonic oscillator or a free particle, and consequently we would decide the value of $\theta(k, q)$, which is impossible.

Therefore, given the family $h_{k}$, there is no algorithm to compute the solution for the associated Hamilton-Jacobi equation, and as a consequence the general problem (for an arbitrary set of quadrature-integrable Hamiltonians) cannot be algorithmically solved, since if it were, we would decide the set $h_{k}$ in the above sense, which is impossible.

The second proof is a variation of the preceding one that, however, does not use $\theta(m)$ (da Costa and Doria, 1990b). Let $h=p_{1}$ and $h^{\prime}=p_{2}^{2}+q_{2}^{2}$. Then put

$$
h_{m}=h+C\left(m, p_{1}\right) h^{\prime}
$$


where $C(m, x)$ is given in Corollary 3.18 , is again a family of integrable Hamiltonians that either represent a single free particle or a free particle uncoupled to a harmonic oscillator. Notice that in the present situation we are dealing with a much more restricted algebra of functions than our $\mathscr{2}$ in Corollary 3.18 .

We have thus settled question 2.

Remark 4.6. Notice that the family $h_{k}$ gives an example of a phenomenon that has been recently pointed out by Moore (1990) in the context of chaotic dynamics: even if we can computably determine with absolute precision a point in the orbit of any of the systems described by $h_{k}$, we have no general algorithmic procedure to determine its trajectory. However, in the present case we are dealing with a family of systems that are nonchaotic and integrable; Turing universality (as in Moore's example) can be realized if our $\theta$ is concocted out of the universal Diophantine equation (da Costa and Doria, 1990b; Davis et al., 1976). Therefore, formal predictability also fails in the "nice" case of integrable systems.

But could we not somehow look at the actual physical system described by an $h_{m}$ and immediately decide whether one has a harmonic oscillator or not? We have proposed elsewhere (da Costa and Doria, 1990b) a thoughtexperiment [which depends on a remark by Scarpellini (1963)] that would allow us to decide the Halting Problem out of a family of systems like the $h_{m}$; it remains to be realized at the concrete level.

We can now turn to the first question: Given an arbitrary Hamiltonian $h$, is there an algorithm that allows us to decide whether the associated Hamiltonian system $X_{h}$ is integrable by quadratures?

The answer is, no. We can offer two different proofs for this negative result. The first proof goes as follows: again let $\mathscr{B}(U)$ be an adequate extension of the algebra of (real-valued) elementary functions on a symplectic manifold $P$, where $U \subset P$ is a star-shaped open Poincaré domain in $P$. Then:

Proposition 4.7. The set $\mathscr{H}(U) \subset \mathscr{B}(U)$ of all elementary Hamiltonian functions on $U$ with a first integral expressed by a quadrature is not recursive.

Remark 4.8. The restriction on the dimension is to allow for the existence of nonintegrable systems (Holmes and Marsden, 1982).

Proof. If $h$ is a Hamiltonian on $U, f$ is one of its first integrals if and only if $\{h, f\}=0$. Therefore, it is easy to show that the set of all such couples is recursively enumerable. We proceed as follows:

1. We restrict our attention to elements of $\mathscr{B}$ with coefficients in the rationals $Q$. Therefore, $\mathscr{B}(U)$ is a countable set and can be effectively enumerated. 
2. The set $\mathscr{F}(U)=\mathscr{B}(U) \cup\left\{\int_{q}^{x} f(\zeta) d \zeta: q \in \mathrm{Q}, f \in \mathscr{B}(U)\right\}$, where $\zeta$ denotes an adequate set of variables on $U$, can also be effectively enumerated.

3. All the operations required for the calculation of the expression for the Poisson bracket $\{h, f\}$ can be effectively described for the formal expressions that represent the elements of both $\mathscr{B}$ and $\mathscr{F}$.

4. We get expressions of the form $B(q, p)$ at the end of the computation of the bracket, which again must be algorithmically checked to see whether one has $B=0$, for all $p, q$, or not.

5. We then generate a recursively enumerable set of pairs $\{h, f\}=0$ by calculating $\{h, f\}_{1}$ one step (the indices refer to the enumeration of the pairs $\langle h, g\rangle) ; \ldots$ the first, second, $\ldots$, ith pairs

$$
\{h, f\}_{1}, \ldots,\{h, f\}_{i}
$$

i times, and add to the set we are thus generating all those that stop before or at that ith step.

Thus, the set of all Hamiltonians on $U$ so that the associated Hamiltonian flows can be integrated by quadratures is recursively enumerable. However, it is not recursive, as there is no enumeration procedure for its complement, due to the negative solution of question 3 .

The second proof is similar to the one that settled question 2: we state:

Proposition 4.9. There is a family $h_{k}, k \in \omega_{0}$, of Hamiltonians in $U$ so that there is no general decision procedure to check, for an arbitrary $k$, whether $X_{h_{k}}$ can be integrated by quadratures.

Proof. Let $h$ be integrable by quadratures and let $h^{\prime}$ not be integrable by quadratures (say, the Holmes-Marsden example, or - if we allow for a higher-dimensional phase space, a 3-body-like problem). We form the linear combination $h_{k}=\theta_{k} h+\left(1-\theta_{k}\right) h^{\prime}$. Then there is no general decision procedure to check whether, given an arbitrary $k$, one deals with a "nice" or "nasty" Hamiltonian.

A variant of this proof could follow the idea in the second proof for our result on question 2 .

Corollary 4.10. There is no general algorithm to decide whether an arbitrary Hamiltonian system over $U$ can be solved by quadratures.

Proof. From the preceding result.

Question 1 is thus dealt with.

Remark 4.11. We also cast a shadow on a quite commonplace assumption made when one studies physical (or any other "concrete" systems) from 
a formal point of view. When formally depicting a scientific structure, say, a physical theory, one usually deals with an object of the form $\Phi=\langle M, \Delta, \rho\rangle$, where $M$ is a formalization of the theory, $\Delta$ is a "domain of applicability," and $\rho$ is a set of correspondence rules. Everything is supposed to be embedded into some set theory, so that we can operate over $\Phi$. Well, we have just proved that $\rho$ cannot be algorithmically defined for physically interesting theories, such as classical mechanics or any other theory where we can repeat the above construction (say, quantum mechanics, or general relativity, or electromagnetism), as we cannot decide whether, say, $h(q, p)$ represents a free particle or a harmonic oscillator, as we showed above.

\subsection{Undecidability of Chaos}

Let $X$ be a smooth vectorfield on a differentiable manifold $M$. Can we algorithmically check whether $X$ has a chaotic behavior-in any of the usual meanings for that word, that is, given an arbitrary vectorfield $X$, can we algorithmically decide whether $X$ is chaotic?

This problem was explicitly discussed by Hirsch (1985) when he makes some remarks about the Lorenz system of equations (Lorenz, 1963):

\footnotetext{
By computer simulation Lorenz found that trajectories seem to wander back and forth between two particular stationary states, in a random, unpredictable way. Trajectories which start out very close together eventually diverge, with no relationship between long run behaviors.

But this type of chaotic behavior has not been proved. As far as I am aware, practically nothing has been proved about this particular system ...

A major challenge to mathematicians is to determine which dynamical systems are chaotic and which are not. Ideally one should be able to tell from the form of the differential equations.
}

(Emphasis added.) Therefore we can ask: Is there a general algorithmic criterion so that, given a definition for chaos in a dynamical system, we can determine whether an arbitrary system satisfies that definition?

Again the answer is, no. Let $M$ be a differentiable manifold, and let $U \subset M$ be a star-shaped open domain. As always, we suppose that ZFC is arithmetically consistent, and that our results happen within ZFC.

We assert:

Proposition 4.12. There is no general algorithmic procedure to check:

1. Whether an arbitrary vectorfield $X$ over $U$ is ergodic.

2. If $\operatorname{dim} M \geq 4$, whether an arbitrary vectorfield $X$ over $U$ has a Smale horseshoe.

3. If $M$ is compact, real, two-dimensional, of class $C^{3}$, and has a constant negative curvature, whether an arbitrary $X$ is a Bernouillian flow. 
Proof. As above. For the first two assertions, let $K_{0}$ be a constant vectorfield on $U$, and let $Y$ be ergodic, or have a Smale horseshoe [for an explicit example, see Holmes and Marsden (1982)]. Now let $\theta$ be the "yesno" function in Corollary 3.18. Then

$$
Z_{m}=\Theta_{m} K_{0}+\left(1-\Theta_{m}\right) Y
$$

where

$$
\Theta_{m}\left(x_{1}, \ldots, x_{n}\right)=\theta_{m}\left(x_{1}\right)
$$

has the same smoothness properties of $Y$, as both $K_{0}$ and $\theta$ are constant functions. It is an undecidable countable family of vectorfields, as in the preceding results.

For the third assertion, we know that (Ornstein and Weiss, 1973) geodesic flows on such an $M$ are Bernouillian. Then, if $X$ is one such flow, we write

$$
Z_{m}=\theta(m) X
$$

Again we cannot in general decide whether we have a trivial zero field or a Bernouillian flow.

\section{GÖDEL'S THEOREM IN CLASSICAL MECHANICS}

Several of the preceding undecidability results can be reformulated as incompleteness theorems within an adequate formalization of classical mechanics and of dynamical systems theory; Zermelo-Fraenkel theory is one such adequate setting for it. We state and prove two incompleteness theorems that stem from what we have done in Section 4.

\subsection{A Hamiltonian Version of Gödel's Theorem}

Notice that all our models $\mathbf{M}$ for the ZFC axioms include the standard model $\mathbf{N}$ for elementary arithmetic as its model for ZFC-arithmetic.

Proposition 5.1. If ZFC is arithmetically consistent, then there is a Hamiltonian system of which it is true in $\mathbf{M}$ that it cannot be integrated by quadratures, but such that this fact cannot be proved within the given axiomatization for Hamiltonian mechanics.

Remark 5.2. We must add to our statement the caveat in Davis (1973, Theorem 7.7). Actually, we only require here an arithmetically consistent axiomatization that is strong enough to contain symplectic geometry and the theory of Diophantine equations, plus all the apparatus of Richardson's functor. 
Proof. A formal proof can be directly obtained out of Proposition 3.28. However, we can offer here an informal argument that still has the flavor of the original argument in Post (1944).

We imitate Davis (1973). We generate all the theorems in the given axiomatization. Within such a listing we form two sublists: list A contains the Hamiltonians whose associated vectorfields can be provably integrated by quadratures. List $A$ is recursively enumerable, we know. List $B$ contains those that we have proved that cannot be integrated by quadratures. Now list B cannot contain all Hamiltonian systems that cannot be thus integrated. For if it did, the set of all those Hamiltonians which cannot be "nicely" integrated would be recursively enumerable, and there would be a decision procedure for integrability. Thus, there is a Hamiltonian in our theory that cannot be integrated by quadratures, but that fact cannot be proved within the given axiomatization.

Proposition 5.1 shows that the difficulty in finding nice integrals for Hamiltonian systems is of the same nature as the incompleteness in Gödel's Theorem. There are things that one simply cannot prove, even if we may suspect that we are dealing with a true fact.

\subsection{Chaos Beyond the Reach of Mathematical Proof}

Our results on chaos can also be formulated as incompleteness results:

Proposition 5.3. If ZFC is arithmetically consistent, then:

1. There is a motion $m(t)$ on $\mathbf{R}^{2}$ of which it is true in $\mathbf{M}$ that $m(t)$ is ergodic in $R^{2}$, but such that its ergodicity cannot be proved from the axioms of the theory.

2. There is a dynamical system of which it is true in $\mathbf{M}$ that it has a Smale horseshoe, but such that the existence of the horseshoe cannot be proved from the axioms of ZFC.

3. There is a flow of which it is true in $\mathbf{M}$ that it is a Bernouillian flow, but such that this fact cannot also be proved from the ZFC axioms to be so.

4. If $\psi$ is a $\mathrm{ZFC}$ predicate that gives a characterization for chaos in a dynamical system, so that ZFC $\vdash \exists x \psi(x)$ and also ZFC $\vdash \exists x \neg \psi(x)$, then there is a dynamical system $Z$ such that $\mathbf{M} \vDash \psi(Z)$, but $\mathrm{ZFC} \nvdash \psi(Z)$ and $\mathrm{ZFC} \nvdash \neg \psi \psi(Z)$.

Proof. Goes on as above; follows directly from Proposition 3.28.

We can also offer an intuitive proof for the first assertion, if we slightly change the argument in Proposition 4.1. We enumerate all open squares 
with rational centers and rational sides parallel to the coordinate axes in the plane $\mathbf{R}^{2}$.

We then make two enumerations, one of the squares with rational centers and sides as described above, which list we denote $S$, and another of all motions $T \subset \mathscr{A}\left(\mathrm{R}^{2}\right)$. We consider all pairs $\langle s, t\rangle_{i}, i \in \omega_{0}, s \in S$ and $t \in T$, and algorithmically start checking whether $t \cap s \neq \varnothing$ or not.

We then start to enumerate all theorems in our theory. We make two listings out of the theorem's list: one, list NE, will include all pairs $\langle s, t\rangle$ so that $t$ does not provably go through $s$. The second one, list $\mathrm{E}$, will include all motions $t \in T$ that are provably ergodic.

Now there must be an ergodic motion $t$ that will not appear in list $\mathrm{E}$. For otherwise we would have a decision procedure for the question "does $t$ goes through $s ?$ ? and that is impossible.

\section{CONCLUSIONS}

We may now summarize the main features of our undecidability results :

1. We have encoded Diophantine problems into classical mechanics, so that the solutions of some algorithmicity questions in mechanics are seen to be Diophantine problems under a disguise.

2. With the help of the Matijaševič-Davis-Robinson universal Diophantine equation (Davis et al., 1976) plus Richardson's explicit construction of an elementary function out of a prescribed Diophantine equation (Richardson, 1968), we can explicitly obtain a function like $G(m, x)$ in Corollary 3.27 .

We can go further, and obtain a function $K(m, x)$ that mirrors the properties of Chaitin's $\Omega$-number (Chaitin, 1988), so that one sees that at worst the undecidability questions we are dealing with behave in an erratic, random way.

3. Moreover, as a consequence of the third assertion in Proposition 3.28 , we can be sure that, even if there are proofs for, say, the integrability of particular Hamiltonian systems, or the existence of chaos in certain families of dynamical systems, such proofs can be arbitrarily long, which means that they can be very difficult.

Especially when one deals with chaos theory, and with the tremendous effort that has been made to ascertain the properties of innocent-looking systems such as the Lorenz system (Hirsch, 1985; Lorenz, 1963), to almost no avail, one wonders if one of those intractability-turned-into-hopelessness effects is at work in such cases.

4. However, there is a bright side in those questions, as new "solvable" Hamiltonian problems can be explicitly constructed out of tractable (algorithmically solvable) families of Diophantine equations. 
We get an actual recipe to do so; it remains to see if that recipe leads to physically sensible Hamiltonian systems.

As Proposition 3.28 shows, undecidability and incompleteness are to be found everywhere in mathematical physics, since the usual function spaces we deal with when modeling physical phenomena all include the algebra of elementary functions.

We must also emphasize related points (P. Suppes, private comments):

1. While we have given general existential proofs concerning undecidability and incompleteness in physics, we have not considered up to now the same questions with respect to particular physically interesting systems. That is to say: if we consider, for instance, the Lorenz system, how much can we know about it? This is certainly a much harder problem. If we follow the approach of the present paper, in order to deal with specific situations one would have to develop a functor like Richardson's from Diophantine equations into the particular theory we are interested in, and then prove it to be undecidable and incomplete out of the similar results for Diophantine equations. Or one would have to prove that such a functor cannot be constructed.

Anyway, dealing with specific situations is assuredly much more difficult than to prove general facts. Yet we believe that the results we have obtained can be also understood as some sort of warning sign : even if it looks obvious that a system has a given property, it does not immediately follow that we can prove it to have that property within a first-order language - we may never be able to.

2. We can also turn the tables around and ask about the physical feasibility of deciding undecidable sentences in a formal theory out of an experiment. That can certainly be done at the Gedanken-experiment level (da Costa and Doria, 1990b; Scarpellini, 1963), despite the fact that its implementation through the simulation of a continuous process may certainly be tricky from the practical viewpoint.

What can we make out of all this? We cautiously suggest that the trouble may lie not in some essential inner weakness or flaw of mathematical reasoning, but in a too narrow, too limited concept of formal system and of mathematical proof. There is a strongly mechanical, machinery-like archetype behind our current formalizations for the idea of algorithmicity that seems to stem from an outdated 17th century vision à la Descartes (even if our current notion of proof is traced back to Green mathematics). Also, a first-order language such as the one for Zermelo-Fraenkel theory is too weak: even if we can prove all of classical mathematics within it, it is marred by the plethora of undecidability and incompleteness results that we can prove about it, and which affect interesting questions that are also relevant for mathematically-based theories such as physics. 
The authors certainly do not know how to, let us say, safely go beyond the limits of the presently available concepts of computability, algorithmicity, and formal system, but they feel that if there are so many quite commonplace things that "should" somehow be provable or decidable within a sensible mathematical structure, and which, however, turn out to be algorithmically undecidable or unprovable, then one cannot blame the whole of mathematics for that. Mathematics is not at fault here. The problem lies in our current ideas about formalized mathematics. They are too weak.

We must look beyond them.

\section{ACKNOWLEDGMENTS}

The questions discussed in the present paper arose out of a series of seminars given by F.A.D. at the University of São Paulo's Institute for Advanced Studies at the invitation of the first author. However, the present paper was only completed at the Institute for Mathematical Studies in the Social Sciences, Stanford University; we owe Prof. Patrick Suppes the indication of Richardson's paper and its relevance to our research, as well as a detailed criticism of two earlier versions of the present paper. We must also thank J. Stolfi from DEC SRC for his BITNET-carried comments on our work, and J. Zimbarg for pleasant discussions, criticism, and observations.

F.A.D. wishes also to thank Prof. Suppes for his hospitality at IMSSS. We acknowledge support from CNPq (Brazil), FAPESP, and the Fulbright Program.

Finally, we must also thank D. Getschko and A. Loparic, who, at the BR-FAPESP node of BITNET, helped smoothen the communication between the authors of this paper.

\section{REFERENCES}

Adler, A. (1969). Proceedings of the American Mathematical Society, 22, 523.

Arnold, V. (1976). Méthodes Mathématiques de la Mécanique Classique, Mir, Moscow.

Blum, L., Shub, M., and Smale, S. (1989). Bulletin of the American Mathematical Society, 21, 1 .

Caviness, B. F. (1970). Journal ACM, 17, 385.

Chaitin, G. J. (1982). International Journal of Theoretical Physics, 22, 941.

Chaitin, G. J. (1988). Algorithmic Information Theory, Cambridge.

Cohen, P. J. (1966). Set Theory and the Continuum Hypothesis, Benjamin, New York.

Da Costa, N. C. A., and Doria, F. A. (1990a). Suppes predicates for classical physics, in Proceedings of the San Sebastiann Congress on Scientific Structures.

Da Costa, N. C. A., and Doria, F. A. (1990b). Non-computability and two conjectures by Penrose and Scarpellini, preprint.

Da Costa, N. C. A., and Doria, F. A. (1991). Structures, Suppes predicates, and Booleanvalued models in physies, in Festschrift in Honor of V. I. Smirnov on His 60th Birthday, J. Hintikka, ed. 
Da Costa, N. C. A., Doria, F. A., and de Barros, J. A. (1990). International Journal of Theoretical Physics, 29, 935.

Dales, H. G., and Woodin, W. H. (1987). An Introduction to Independence for Analysts, Cambridge.

Davenport, J. H. (1981). On the Integration of Algebraic Functions, Springer Lecture Notes in Computer Science \#102.

Davis, M. (1973). American Mathematics Monthly, 80, 233.

Davis, M., Matijaševič, Yu., and Robinson, J. (1976). Hilbert's Tenth Problem. Diophantine equations: Positive aspects of a negative solution, in Mathematical Developments Arising from Hilbert Problems, F. E. Browder, ed., Proceedings of the Symposium on Pure Mathematics, Vol. 28.

Davis, M., Putnam, H., and Robinson, J. (1961). Annals of Mathematics, 74, 425.

Ehrenfeucht, A., and Mycielski, J. (1971). Bulletin of the American Mathematical Society, 17, 366.

Gödel, K. (1931). Monatshefte für Mathematik and Physik, 38, 173.

Hirsch, M. (1985). The chaos of dynamical systems, in Chaos, Fractals and Dynamics, P. Fischer and W. R. Smith, eds., Marcel Dekker.

Holmes, P. J., and Marsden, J. (1982). Communications in Mathematical Physics, 82, 523.

Jantscher, L. (1971). Distributionen, W. de Gruyter.

Jones, J. P. (1982). Journal of Symbolic Logic, 47, 549.

Jones, J. P., and Matijaševič, Yu. (1984). Journal of Symbolic Logic, 49, 818.

Kunen, K., and Vaught, J. E. (1984). Handbook of Set-theoretic Topology, North-Holland.

Lichtenberg, A. J., and Lieberman, M. A. (1983). Regular and Stochastic Motion, Springer.

Lorenz, E. (1963). Journal of Atmospheric Science, 20, 130.

Machtey, M., and Young, P. (1978). An Introduction to the General Theory of Algorithms, North-Holland.

Manin, Yu. I. (1977). A Course in Mathematical Logic, Springer.

Mendelson, E. (1987). Introduction to Mathematical Logic, 3rd ed., Wadsworth \& Brooks.

Moore, C. (1990), Physical Review Letters, 64, 2354.

Ornstein, D., and Weiss, B. (1973). Israel Journal of Mathematics, 14, 184.

Post, E. (1944). Bulletin of the American Mathematical Society, 50, 284.

Pour-El, M. B., and Caldwell, J. (1975). Zeitschrift für Mathematische Logik und Grundlagen der Mathematik, 21, 1.

Pour-El, M. B., and Richards, I. (1981). Advances in Mathematics, 39, 215.

Pour-El, M. B., and Richards, I. (1983a). Advances in Mathematics, 48, 44.

Pour-El, M. B., and Richards, I. (1983b). Transactions of the American Mathematical Society, $275,539$.

Pour-El, M. B., and Richards, I. (1989). Computability in Analysis and Physics, Springer.

Richardson, D. (1968). Journal of Symbolic Logic, 33, 514.

Richardson, D. (1969). Zeitschrift für Mathematische Logik und Grundlagen der Mathematik, $15,333$.

Rogers, Jr., H. (1967). Theory of Recursive Functions and Effective Computability, McGrawHill, New York.

Scarpellini, B. (1963). Zeitschrift für Mathematische Logik und Grundlagen der Mathematik, 9, 265.

Şierpiński, W. (1956). Hypothèse du Continu, Chelsea Press, New York.

Tabor, M. (1989). Chaos and Integrability in Nonlinear Dynamics, Wiley, New York.

Uspensky, V. A. (1987). Gödel's Incompleteness Theorem, Mir, Moscow.

Wang, P. (1974). Journal ACM, 21, 586.

Yosida, K. (1980). Functional Analysis, Springer. 